\title{
Alterations of kinematics in knees after single versus multiple radius femoral prostheses total knee arthroplasty: a retrospective study
}

Mengyuan $\mathrm{Li}^{1 \dagger}$, Guangtao $\mathrm{Fu}^{1+}$, Wenhan Huang ${ }^{1 \dagger}$, Bofu Lin ${ }^{1,2}$, Ruiying Zhang ${ }^{1}$, Yu Zhang ${ }^{1}$, Yuanchen Ma ${ }^{1 *}$ and Qiujian Zheng ${ }^{1 *}$ (D)

\begin{abstract}
Background: Design modifications in prostheses may cause alterations in gait kinematics, thus influencing functional restoration of knees after total knee arthroplasty (TKA). The aim of the study was to investigate the differences in gait kinematics and clinical outcomes after single radius (SR) versus multiple radius (MR) TKA.
\end{abstract}

Method: The present retrospective study included 38 unilateral TKA involving 20 knees using MR design implant and 18 knees using SR design implant. Thirty-six healthy volunteers were also recruited. The mean follow-up time was $16 \pm 3$ months. At the end of follow-up, the 6 degrees of freedom (DOF) kinematics of knees and range of motion (ROM) were measured with a portable optical tracking system. Knee society score (KSS) and knee injury, and osteoarthritis outcome score (KOOS) were also collected.

Results: Patients in the SR group had significantly higher scores in activities of daily living $(84.7 \pm 15.9)$ and sports and recreation (67.5 \pm 25.2$) \mathrm{KOOS}$ sub-score than MR group ( $69.9 \pm 17.6, P=0.012 ; 50.0 \pm 20.8, P=0.027$, respectively). Significant differences were detected between MR knees and SR knees $\left(1.82^{\circ} \pm 3.11^{\circ}\right.$ vs $\left.4.93^{\circ} \pm 3.58^{\circ}, P=0.009\right)$, and MR knees and healthy knees $\left(1.82^{\circ} \pm 3.11^{\circ}\right.$ vs $\left.3.62^{\circ} \pm 3.52^{\circ}, P=0.032\right)$ in adduction/abduction ROM. The proximal/distal translation was significantly smaller in MR knees $(0.58 \pm 0.54 \mathrm{~cm})$ compared with SR knees $(1.03 \pm 0.53 \mathrm{~cm}, P=0.003)$ or healthy knees $(0.84 \pm 0.45 \mathrm{~cm}, P=0.039)$. SR knees $(0.24 \pm 0.40 \mathrm{~cm})$ had smaller translation compared with the MR group $(0.54 \pm 0.33 \mathrm{~cm}, P=0.017)$ and control group $(0.67 \pm 0.36 \mathrm{~cm}, P=0.028)$. No significant difference was detected in the other DOFs during the gait cycle. Significant difference was detected in extension/flexion, internal/external rotation, adduction/abduction, proximal/distal and medial/lateral among MR, SR and healthy knees.

Conclusion: After TKA, patients have altered gait kinematics compared with the control group. MR and SR design showed varied characteristics in 6 DOF gait kinematics, which could be the cause of the difference in functional outcome.

Keywords: Total knee arthroplasty, Single radius, Multiple radius, Gait kinematics

\footnotetext{
*Correspondence: myc0998@gmail.com; zqj650@126.com

${ }^{+}$Mengyuan Li, Guangtao Fu and Wenhan Huang contributed equally to this work.

'Division of Joint Osteopathy and Traumatology, Center of Orthopedics Surgery, Guangdong Provincial People's Hospital, Guangdong Academy of Medical Sciences, School of Medicine, South China University of Technology, 106 Zhongshaner Road, Yuexiu District, Guangzhou 510080, PR China

Full list of author information is available at the end of the article
}

(C) The Author(s). 2020 Open Access This article is licensed under a Creative Commons Attribution 4.0 International License, which permits use, sharing, adaptation, distribution and reproduction in any medium or format, as long as you give appropriate credit to the original author(s) and the source, provide a link to the Creative Commons licence, and indicate if changes were made. The images or other third party material in this article are included in the article's Creative Commons licence, unless indicated otherwise in a credit line to the material. If material is not included in the article's Creative Commons licence and your intended use is not permitted by statutory regulation or exceeds the permitted use, you will need to obtain permission directly from the copyright holder. To view a copy of this licence, visit http://creativecommons.org/licenses/by/4.0/ The Creative Commons Public Domain Dedication waiver (http://creativecommons.org/publicdomain/zero/1.0/) applies to the data made available in this article, unless otherwise stated in a credit line to the data. 


\section{Background}

The number of total knee arthroplasty (TKA) is expected to grow exponentially in the coming years, because this procedure is effective in relieving pain and improve function in patients with end-stage osteoarthritis of the knee [1-3]. Nonetheless, approximately 20\% of patients remained dissatisfied with the outcome [4]. Limited range of motion, anterior knee pain, instability and extensor insufficiency are among the leading postoperative complaints [4-6]. In vivo kinematics of the knee after TKA are alternated, and thus are probably the cause. Therefore, there has been a recent increase in recognition of the importance of the prostheses' kinematics in accordance to the natural knee.

In the classical knee kinematics, there exist at least two transient rotating centers in the knee within the functional knee range of motion. When the femoral condyle moves downward and backward during flexion, the radius of the condylar curvature diminishes [7]. Thus, most of the femoral components of contemporary TKA are designed as multi-radius (MR). On the other hand, Eckhoff [8] reported that MR curves and changing centers of the posterior femur condyles were presented while observing from the traditional coronal, sagittal, and transverse planes. When viewing in the plane perpendicular to the transepicondylar axis, the posterior condyles of the femur were curves with single curvature radius [9]. This cylindrical axis was coincident with the natural flexion-extension axis of the knee, passing through the origins of the anterior cruciate ligament and posterior cruciate ligament, which had been confirmed by kinematics studies [7,9]. Therefore, some femoral component designs have incorporated a single radius.

Clinical studies and meta-analysis that compared the SR and MR femoral design based on the clinical scores revealed contradictory results $[4,10-15]$. The probable reason is that clinical scores may not be sensitive enough to elucidate outcome differences in implant designs. In order to highlight differences between knee prosthetic designs, demanding functional tasks are required. Our previous isokinetic and isometric data showed that SR design had advantages on higher extension and flexion strength than MR design [16]. However, knees are complex joints providing function and proprioception within 6 degrees of freedom (DOF) [17-19]. The effects of curvature modifications in design on daily activities still remain indeterminate. Walking is the most frequent activity of the daily life even for patients who had TKA. In the present study, we conducted gait analysis to assess knee kinematics during level walking. The purpose is to test the hypothesis that the kinematic behavior of the TKA knees varied with the femoral prostheses design, measuring the 6 DOF kinematics of MR or SR TKA knees.

\section{Methods}

This retrospective, comparative study design was approved by the Institutional Review Board of our hospital. We obtained signed informed consent for participation from all study patients.

Inclusion criteria were as follows: (1) primary symptomatic osteoarthritis of the knee, patient with asymptomatic but degenerative contralateral knee was also included; (2) 55 to 85 years old; (3) body mass index (BMI) lower than $35 \mathrm{~kg} / \mathrm{m}^{2}$; (4) American Society of Anesthesiologists (ASA) class 1 or 2; (5) follow-up period longer than 1 year. Exclusion criteria were as follows: (1) inflammatory arthritis, including rheumatoid arthritis, suppurative arthritis or gouty arthritis; (2) revision TKA; (3) previous tibial or femoral osteotomy; (4) flexion contracture or extension deficit more than $10^{\circ}$; (5) varus or valgus malalignment more than $10^{\circ}$; or (6) any other lower extremity disease, including tumor, infection, etc..

Knee Society Score (KSS) and Knee Injury, and Osteoarthritis Outcome Score (KOOS) were collected to assess the subjective knee function. In order to compare gait kinematics between TKA knees and normal knees, we also recruited 36 healthy people. These volunteers met the following criteria: (1) no symptomatic osteoarthritis of the knee; (2) no lower extremity deformity; (3) no other lower extremity disease, including tumor, infection, injury or history of surgery. A portable optical marker-based motion analysis system (Opti_Knee; Innomotion Inc., Shanghai, China) was utilized to measure the 6 DOF kinematics of the SR TKA knees, MR TKA knees and healthy knees during treadmill gait (Fig. 1a). The range of motion (ROM) of each DOF in the entire gait cycle was calculated. Then, comparison of 6 DOF kinematics was performed between SR and MR TKA knees. We also compared the 6 DOF kinematics between either SR or MR TKA knees and healthy knees.

The same experienced surgeon (YCM) performed the surgery. After general or spinal anesthesia and a standard pro-operative antibiotic prophylaxis, all TKAs were performed under tourniquet. The knee was exposed with a straight ventral skin incision and then a straight medial parapatellar capsular approach. The patella was not resurfaced. We applied posterior cruciate ligament (PCL)substituting design for all cases. Measured resection technique was manually performed to achieve appropriate component alignment and patella tracking. A slight tibia posterior slope was set at $3^{\circ}$. For the SR group, the Stryker Triathlon TKA system (Stryker Orthopaedics, Mahwah, New Jersey) was used, and PFC sigma (DePuy Orthopaedics, Inc. Warsaw, IN, USA) was used in the MR group. On the second day, patients in both groups were encouraged to mobilize including full-weight bearing. Other post-operative exercises included continuous passive motion, active of passive knee extension. 


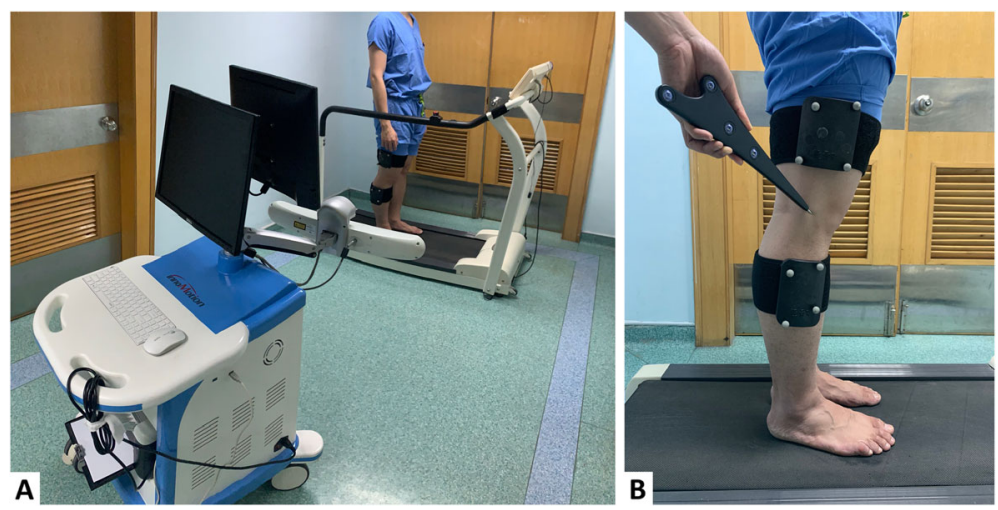

Fig. $\mathbf{1}$ a The instrument for knee kinematics analysis. $\mathbf{b}$ Identification of surface markers

Gait kinematic data was collected following a previously published protocol [17, 19]. Two rigid bodies, which comprise four infrared light-reflecting markers each (Opti_Knee; Innomotion Inc., Shanghai, China), were respectively tight onto patient's thigh and shank with bandages. A handled digitizing probe with four infrared light-reflecting markers was used to identify greater trochanter, lateral epicondyle, medial epicondyle, lateral tibia plateau, medial tibia plateau, medial malleolus, lateral malleolus as the femoral and tibial landmarks at a static phase (Fig. 1b). Based on skeletal landmarks in the system, the femur coordinate system was built by the following steps: (1) The midpoint of the transepicondylar axis was defined as the femoral center, and a line connected the landmarks of the medial and lateral femoral epicondyles; (2) the transepicondylar line was also defined as the medial-lateral axis; (3) the anterior-posterior axis was perpendicular to the plane that was defined by the transepicondylar line and the greater trochanter; (4) the proximal-distal axis was perpendicular to the medial-lateral axis and the anteriorposterior axis. By the similar steps, we built the tibia coordinate system: (1) the center of the tibia coordinate system was located at the midpoint of the line connecting the most medial and lateral points of the tibial plateau; (2) the medial-lateral tibial plateau line was also defined as the medial-lateral axis; (3) the anteriorposterior axis was perpendicular to the plane that was defined by the medial-lateral tibial plateau line and the lateral malleolus; (4) the proximal-distal axis was perpendicular to the medial-lateral axis and the anteriorposterior axis. The rotation was defined as the orientational changes of the tibial coordinate system relative to the femur coordinate system along the anterior-posterior, medial-lateral and proximal-distal axis in the Euler angle sequence, including flexion $(+) /$ extension, internal/external $(+)$ rotation, adduction/abduction $(+)$. Likewise, translation was defined as the displacement of the center of the tibial coordinate system relative to the femur coordinate system, including anterior $(+) /$ posterior translation, proximal/distal $(+)$ translation and medial/lateral $(+)$ translation (Fig. 2). After a 5-min treadmill gait warm-up, the patient walked on the treadmill at a comfortable speed which resembled level-walking pattern. Subsequently, the knee was imaged for $15 \mathrm{~s}$ at a frame rate of $60 \mathrm{~Hz}$. We applied a low-pass filter to smooth the raw kinematics data at $6 \mathrm{~Hz}$, and then knee kinematics was computed and described as rotation and translation. A custom-developed MATLAB (MathWorks Inc) program was used to normalize the gait cycle from a heel strike of $0 \%$ to the next heel strike of $100 \%$ to represent a classical gait cycle. The gait cycle consists of a stance phase (0 to $62 \%$ ) and a swing phase (63 to $100 \%$ ). The stance phase was analyzed in 3 portions: the loading response (early stance), 0 to $12 \%$ of the gait cycle; the mid-stance, 13 to $52 \%$ of the gait cycle; and the terminal stance, 53 to $62 \%$ of the gait cycle. The swing phase was also analyzed in 3 portions: initial swing, 62 to $75 \%$ of the gait cycle; mid-swing, 76 to $85 \%$ of the gait cycle; and terminal swing, 86 to $100 \%$ of the gait cycle [17]. GraphPad Prism 8 (GraphPad Software, Inc., USA) was utilized to generate the ensemble average curve of each DOF.

\section{Statistical analysis}

The data was described as Means and standard deviations. Kolmogorov-Smirnov test was used for testing normality. Chi-square test and Fisher's exact test were used for categorical variables. The range of motion (ROM) in 6 DOF, KSS, KOOS were analyzed by 1-way analysis of variance (ANOVA), and the level of statistical significance was set at $P<0.05$. The least-significantdifference (LSD) test was performed between groups when significant differences were detected. These statistical analyses were done using SPSS 26.0 (SPSS Inc., Chicago, IL, USA). A post-hoc power calculation was determined by the statistical power analyses G Power 3.1 to eliminate type II error. 


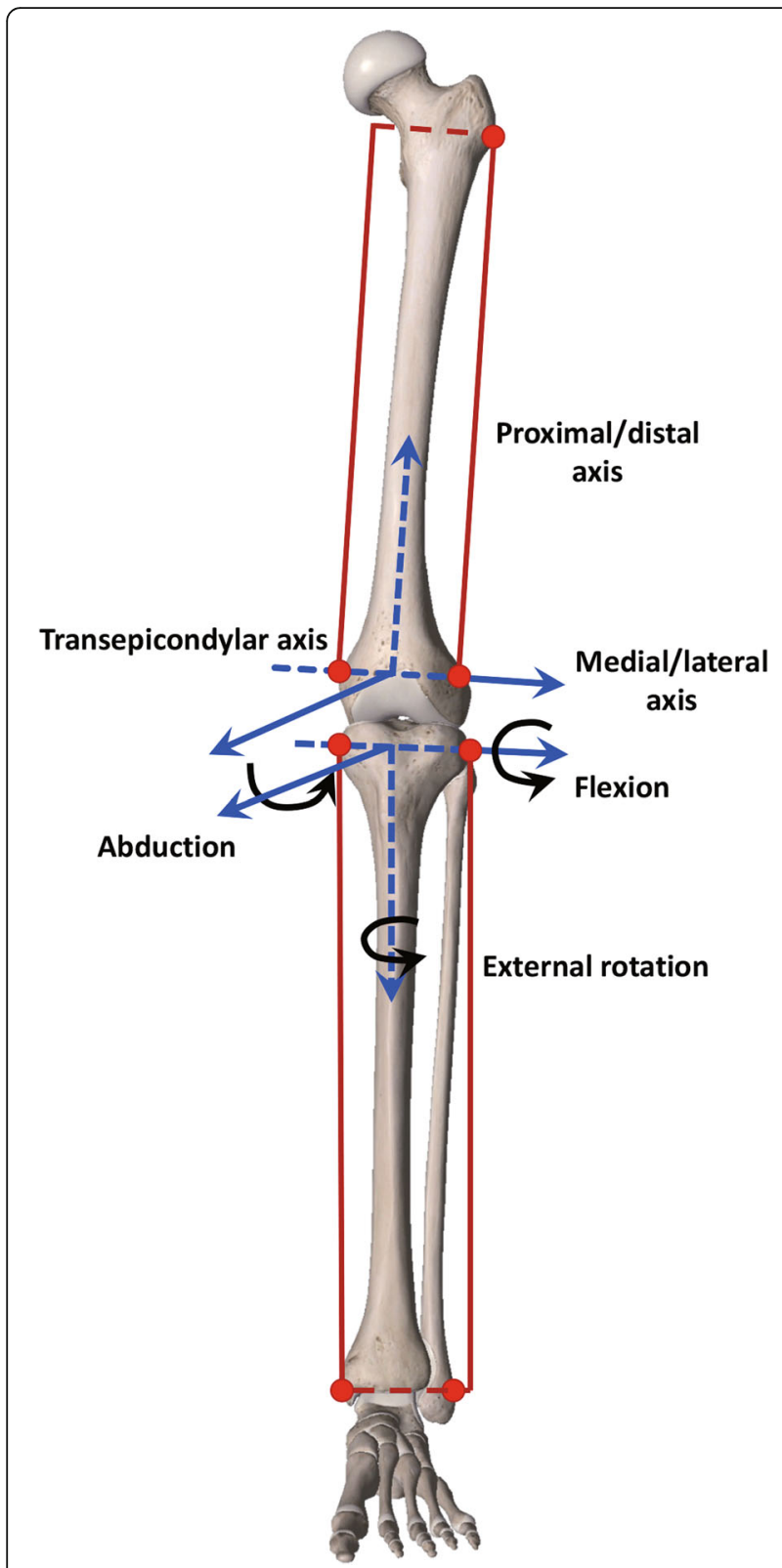

Fig. 2 Definition of local femoral and tibial coordinate systems

\section{Results}

\section{Demographics and clinical evaluation of two groups}

A total of 38 patients underwent unilateral TKA were included in this study (MR group, $n=20$; SR group, $n=$ 18). The mean follow-up time was $16 \pm 3$ months. MR group and SR group had comparable demographic characteristics including sex, age, height, weight, body mass index, surgical side and follow-up time. The data of the two groups were considered homogeneous (Table 1). In post-hoc power calculation, the minimum $\alpha$ power was 0.75. Considering the small sample size of this study, we accepted $\alpha$ power $>0.75$ for detecting a significant difference.
Table 1 Demographic and clinical data (mean \pm SD) of included patients

\begin{tabular}{llll}
\hline & MR group $(n=20)$ & SR group $(n=18)$ & $P$ value \\
\hline Age, years & $65.6 \pm 5.8$ & $62.7 \pm 4.3$ & 0.753 \\
Height, $\mathrm{m}$ & $1.58 \pm 0.06$ & $1.60 \pm 0.04$ & 0.259 \\
Weight, $\mathrm{kg}$ & $71.44 \pm 13.13$ & $63.64 \pm 16.31$ & 0.362 \\
Body mass index & $23.55 \pm 6.93$ & $26.31 \pm 5.72$ & 0.702 \\
Female: male & $16: 4$ & $15: 3$ & 1.000 \\
Right: Left TKA, $\mathrm{n}$ & $11: 9$ & $7: 11$ & 0.321 \\
Follow-up time, months & $17 \pm 4$ & $15 \pm 3$ & 0.293
\end{tabular}

$S D$ Standard deviation, $S R$ Single radius, MR Multiple radius, TKA Total knee arthroplasty

In terms of functional scores, the SR group scored significantly better in activities of daily living $(84.7 \pm 15.9)$ and sports / recreation $(67.5 \pm 25.2)$ KOOS sub-score post-operatively compared with MR group (69.9 \pm 17.6 , $P=0.012 ; \quad 50.0 \pm 20.8, \quad P=0.027$, respectively). There were no significant differences between the two groups in other clinical outcome categories (Table 2 and Fig. 3).

\section{Range of motion and kinematics}

Table 3 and Fig. 4 summarize the range of motion, translation and gait curve for MR, SR and healthy control groups.

In the sagittal plane, there was no significant difference in ROM during gait cycle among MR, SR and healthy group. In regard of gait curve, significant difference was presented in MR and SR during the second half of midstance phase and terminal stance phase (MR: 44\% 58\%, SR: $42 \sim 60 \%$, respectively) compared with healthy knees (Fig. 4a).

In the axial plane, no significant difference in ROM was detected among MR, SR knees and healthy knees. Nonetheless, in the aspect of gait kinematics, MR knees showed more externally rotation during the majority of gait cycle $(0 \sim 12 \%, 36 \sim 60 \%, 92 \sim 100 \%)$ than SR knees.

Table 2 KSS and KOOS (mean \pm SD) after TKA

\begin{tabular}{llll}
\hline & MR group & SR group & $P$ value \\
\hline KSS & & & \\
Knee & $72.0 \pm 20.7$ & $82.1 \pm 14.4$ & 0.099 \\
Function & $75.2 \pm 22.3$ & $79.3 \pm 23.8$ & 0.595 \\
KOOS & & & \\
1 - Pain & $75.6 \pm 13.1$ & $85.1 \pm 16.4$ & 0.057 \\
2 - Symptom & $75.0 \pm 14.6$ & $78.1 \pm 18.1$ & 0.561 \\
3 - Activities of daily living & $69.9 \pm 17.6$ & $84.7 \pm 15.9$ & 0.012 \\
4 - Sports/recreation & $50.0 \pm 20.8$ & $67.5 \pm 25.2$ & 0.027 \\
5 - Quality of life & $57.5 \pm 19.7$ & $67.4 \pm 19.4$ & 0.133 \\
\hline
\end{tabular}

KSS Knee Society Scores,

KOOS Knee Injury, and Osteoarthritis Outcome Score, SD Standard deviation, TKA Total knee arthroplasty, SR Single radius, MR Multiple radius 

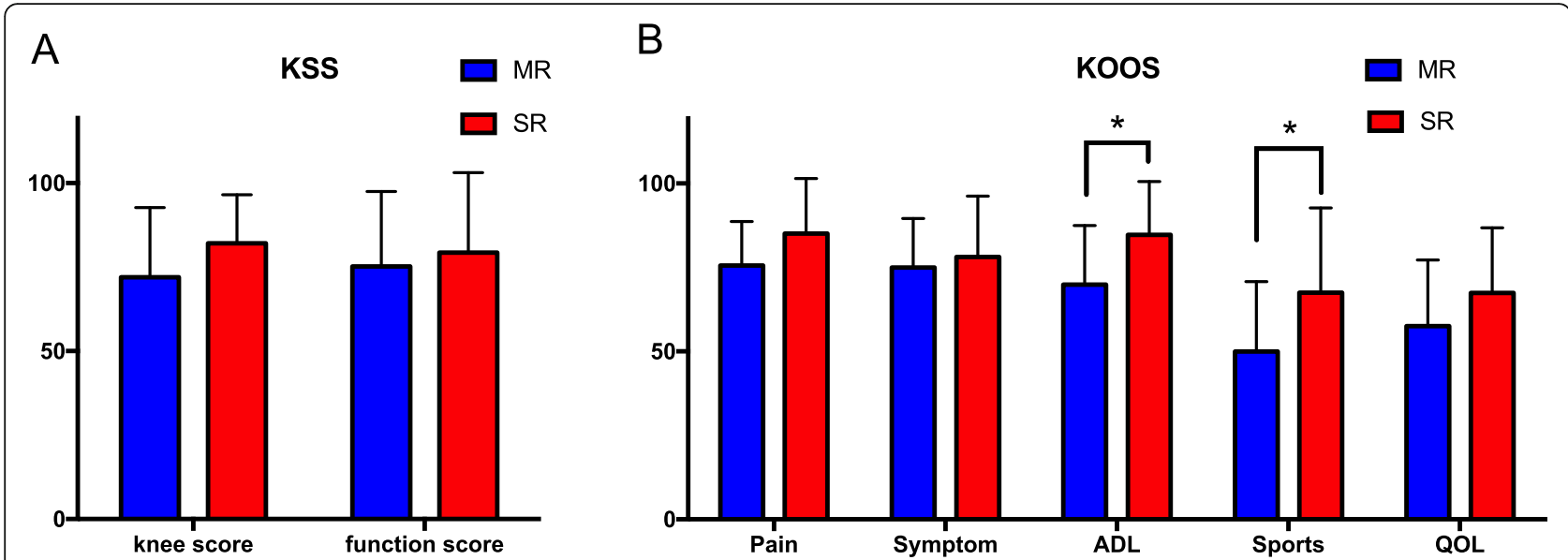

Fig. 3 Functional outcome after TKA. Error bars denote the standard deviation of each group. Segments with significant statistical differences (*: $P<0.05)$ between the groups were marked with asterisks. (TKA: total knee arthroplasty; SR: single radius; MR: multiple radius; KSS: Knee Society Scores, KOOS: Knee Injury, and Osteoarthritis Outcome Score; ADL: activities of daily living; QOL: quality of life)

Similarly, MR knees rotated more externally during the response phase $(0 \sim 8 \%)$ and terminal swing phase $(90 \sim$ $100 \%)$ compared with normal knees. However, during the mid-swing phase (76 84\%), MR knees exhibited an internally rotating trend, while SR knees rotated in an opposite direction. SR knees showed less external rotation than healthy knees during $68 \sim 80 \%$ and less internal rotation $94 \sim 100 \%$ of gait cycle (Fig. $4 \mathrm{c}$ ).

In the coronal plane, significant differences were detected between MR knees and SR knees $\left(1.82^{\circ} \pm 3.11^{\circ}\right.$ vs $4.93^{\circ} \pm$ $\left.3.58^{\circ}, P=0.009\right)$, and MR knees and healthy knees $\left(1.82^{\circ} \pm\right.$ $3.11^{\circ}$ vs $\left.3.62^{\circ} \pm 3.52^{\circ}, P=0.032\right)$. In the gait curve, MR knees presented more abducted mainly in stance phase than SR ( $8 \sim 52 \%)$ and normal ( $8 \sim 60 \%)$ knees (Fig. 4e).

Interestingly, although healthy knees were found to have a more obvious anterior-posterior translation $(0.54 \pm 0.60 \mathrm{~cm})$ compared with the MR group $(0.51 \pm$ $0.43 \mathrm{~cm}, P=0.003)$ and SR group $(0.36 \pm 0.54 \mathrm{~cm}, P=$ 0.039), no significant difference was detected. Moreover, no significant difference was found with regard to the gait curve (Fig. 4b).
As for proximal/distal DOF, significantly smaller translation was observed between MR knees and SR knees $(0.58 \pm 0.54 \mathrm{~cm}$ vs $1.03 \pm 0.53 \mathrm{~cm}, P=0.017)$, and MR knees and healthy knees $(0.58 \pm 0.54$ vs $0.84 \pm$ $0.45 \mathrm{~cm}, P=0.028)$. In the gait curve, MR knees showed significantly less stretched than SR $(78 \sim 84 \%)$ and normal knees $(78 \sim 88 \%)$ in the mid-swing phase (Fig. 4d).

In medial/lateral DOF, SR knees were observed to have a smaller translation (MR: $0.54 \pm 0.33 \mathrm{~cm}$; SR: $0.24 \pm 0.40 \mathrm{~cm}$; Healthy: $0.67 \pm 0.36 \mathrm{~cm}$, respectively). When looking in the gait curve, the significant difference presented between TKA knees and healthy knees. For SR knees, they were significantly more medial shifted during either mid-stance phase $(32 \sim 40 \%)$ and midswing phase $(72 \sim 84 \%)$ in comparison with healthy knees. MR knees showed significantly more medial shifted during $32 \sim 48$ and $80 \%$ of the gait cycle than normal knees, while during the early swing phase (62 $68 \%)$ phase, MR knees were found more lateral translated than normal (Fig. 4f).

Table 3 ROM (mean \pm SD) among MR TKA, SR TKA and healthy knees groups

\begin{tabular}{lllllll}
\hline Parameters & MR & SR & Healthy & & & \\
\cline { 5 - 7 } & & & & MR vs SR & MR vs healthy & SR vs healthy \\
\hline Extension/Flexion ROM $\left(^{\circ}\right)$ & $48.10 \pm 9.03$ & $44.23 \pm 10.54$ & $47.32 \pm 7.60$ & 0.489 & 0.154 & 0.612 \\
Internal/External rotation ROM $\left(^{\circ}\right)$ & $3.86 \pm 2.33$ & $3.41 \pm 2.40$ & $5.37 \pm 4.35$ & 0.707 & 0.247 & 0.228 \\
Adduction/Abduction ROM $\left(^{\circ}\right)$ & $1.82 \pm 3.11$ & $4.93 \pm 3.58$ & $3.62 \pm 3.52$ & 0.009 & 0.032 & 0.781 \\
Anterior-posterior translation (cm) & $0.51 \pm 0.43$ & $0.36 \pm 0.54$ & $0.54 \pm 0.60$ & 0.189 & 0.854 & 0.312 \\
Proximal-distal translation (cm) & $0.58 \pm 0.54$ & $1.03 \pm 0.53$ & $0.84 \pm 0.45$ & 0.003 & 0.039 & 0.525 \\
Medial-lateral translation (cm) & $0.54 \pm 0.33$ & $0.24 \pm 0.40$ & $0.67 \pm 0.36$ & 0.017 & 0.547 & 0.028 \\
\hline
\end{tabular}

SD Standard deviation, TKA Total knee arthroplasty, SR Single radius, MR Multiple radius, ROM Range of motion 


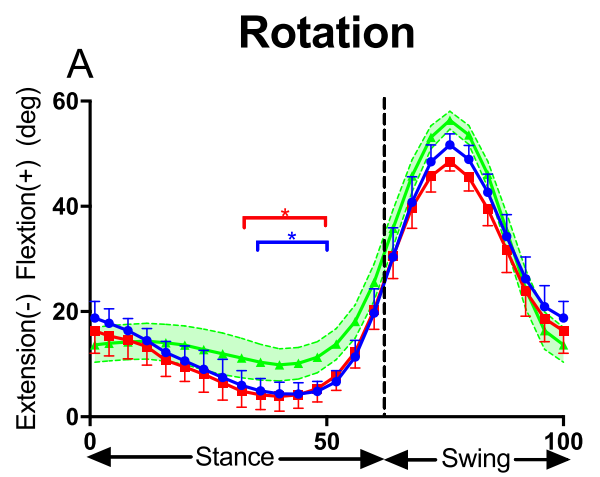

\section{B \\ Translation}
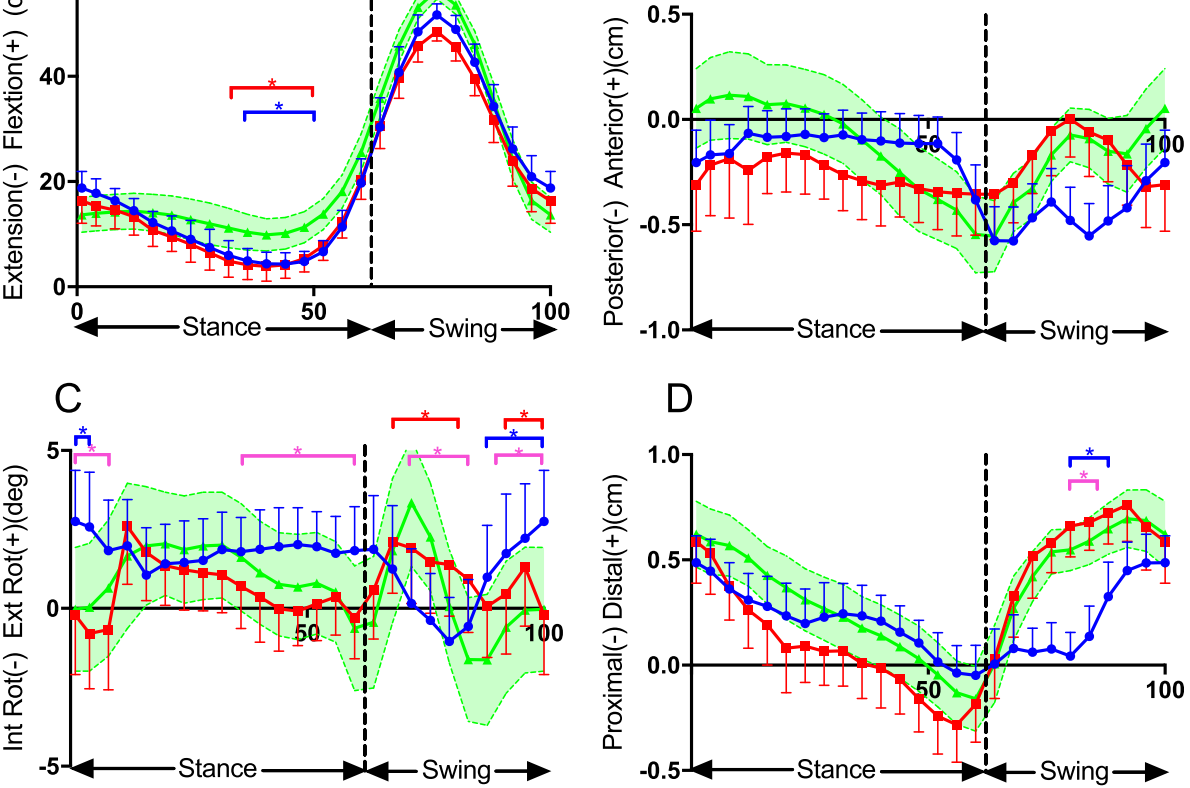

E



$\mathrm{D}$

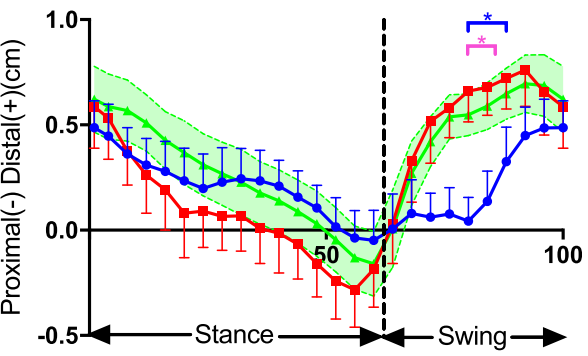

$\mathrm{F}$
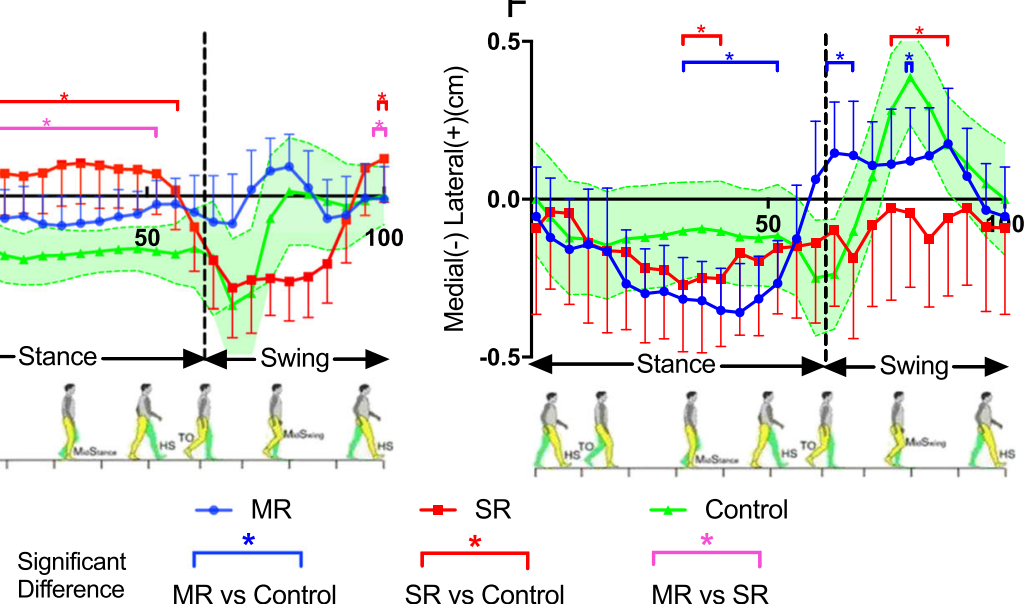

Fig. 4 Six degree of freedom joint kinematics of control, SR, and MR knees. Average curves of knee kinematics with error bars/shadow displaying standard deviation of each group during a gait cycle. Segments with significant statistical differences (*: $P<0.05)$ between the groups were marked with asterisks. (SR: single radius; MR: multiple radius; HS: heel strike; TO: toe off)

\section{Discussion}

According to current literature, quadriceps strength and range of motion during gait decreased in patients undergoing TKAs, but other kinematic parameters such as axial rotation and anterior-posterior translation during gait were seldom analyzed because of limitations in traditional gait analysis techniques [11, 15, 20-22]. The results of our current study support the initial hypothesis that different femoral design in TKA knees can lead to varying kinematics in vivo. It is also corresponded to the clinical evaluation that patients in SR group had significantly higher scores in activities of daily living and sports
/ recreation KOOS sub-score than those in the MR group at the end of the follow-up.

The movements in the sagittal plane, the predominant motion during gait cycle, have been studied previously, and the flexion angles after PCL substituting TKAs during gait were decreased compared with the flexion angle of the normal [11, 15]. Nonetheless, researches compared sagittal ROM of MR and SR had contradictory results $[10,11,15]$. In this context, TKA knees, either MR or SR, had higher extension compared with control group. This could be attributed to the anterior bowing of the femur and the tibial posterior slope. As a result, 
the femoral and tibial components are in approximately $5^{\circ}$ to $10^{\circ}$ of hyperextension relative to the sagittal mechanical axis. Additionally, anterior cruciate ligament (ACL) is to limit anterior tibial translation when the knee is close to extension, so in TKA patients without ACL, they appeared to overcorrect the extended postures during the weight acceptance phase of the gait cycle in order to reduce the functional absence of ACL [23, 24].

Kinematic alterations were also identified in other DOFs in TKA knees. In the transverse plane, SR knees showed an external rotation trend within flexion. This result was in accordance with the results reported by Tamaki $[15,25]$. They reported that from extension to $60^{\circ}$ knee flexion, SR knee presented a medial pivot kinematic pattern, wherein the lateral condyle moved posteriorly significantly compared with the lesser amount of anterior-posterior translation of the medial condyle. This pivot pattern was similar to that reported in normal knee [26, 27]. Additionally, Kessler [28] discovered that the SR design showed finite helical axes concentrated on a single axis near to the medio-lateral axis of the femoral component. However, the MR design showed larger angular and spatial localization deviation, exhibiting finite helical axes varying between two axes.

With respect to the coronal plane, we found that SR knees adducted while flexion, which was close to the mode of healthy knees, but MR knees showed an opposite movement. We suspected that this phenomenon probably related to the changing radius of the femoral prostheses might change the tension on the collateral ligaments and other soft tissues at different points in the range of motion [21, 29]. So, in MR knee, the changing radius of condyle would be more likely to cause a valgus/varus rotation of the femur on the tibia, leading a perception of instability. Conversely, the SR configuration maintains the collateral ligaments in an isometric pattern during knee movement, thereby providing sustained stability.

Overall, TKA knees did not manifest a laxity more than $5 \mathrm{~mm}$ during active movement. We inferred that the cam-post design of tibial insert constrained the anterior translation. In the stance phase, the tibia moved posteriorly relative to femur. In the swing phase, the tibial component of SR knees tended to move posteriorly relative to femur by gravity because the tibial resection was tilted posteriorly [30]. However, in MR design the smaller radius of the femoral component at flexion angles may result in a less constrained effect in anteriorposterior direction, and furtherly lead to slight anterior translation of the femoral component [15].

As far as proximal/distal and medial/lateral translation in TKA knees, there were no such reports in the current literature. In our research, the proximal/distal translation may reflect the change of the joint gap. The compressive loading of the knee during stance phase reduced proximal/distal translation, which was consistent in two designs. In the swing phase, the joint was stretched by gravity, so the translation increased, but it was not so prominent in MR knees. This may indicate a compensation strategy that a stronger co-contraction of muscles to stabilize in the presence of mid-flexion subluxation in MR while the curvature radius changes. The range of medial/lateral translation of SR knees is less fluctuated. We considered this as another implication of the stability and isometry of collateral ligaments. It is noticed that the range of both of MR and SR groups was smaller than the control group, and it might be attributed to the lower compliance of the soft tissue around the joint after TKA.

In the current study, the difference of biomechanics parameters was in line with the difference in patientreported outcome measures. As was discussed previously, SR knees showed better stability in the DOF of internal/external rotation, valgus/varus rotation and proximal/distal translation when comparing with $M R$ knees. Therefore, SR knees showed higher ability in performing more exquisite activities, such as jumping, twisting and jogging. We inferred that this may be the reason for the difference in activities of daily living and sports / recreation KOOS sub-score. Similarly, Cook [12] compared 426 cases of SR and 113 cases of MR designs at 3.9-year mean follow-up and reported that the SR group had a significantly better KSSs, flexion, stability, pain, gait, and stair climbing. Sumner [31] reported that SR TKA knees had more improved kinematic during stair descent than MR TKA knees, which might reflect in the higher KSS-Function score.

This study had some limitations. First, we only included the posterior stabilized and fixed-bearing knee designs. Other designs, such as in cruciate retaining or mobilized platform ones, may mitigate the effect of radius change. Khasian [32] reported that the latest MR design, G-curve femoral prostheses, could improve stability and reduce mid-flexion paradoxical anterior sliding, but this prostheses was not included in the current study. Secondly, the small sample size of 38 and the non-consistent follow-up time point may confound the interpretation. So, a larger-sized, multi-center, long-term study is on the necessity in the future. Third, we opted for a control group of healthy subjects instead of comparing the data between the operated knee and the contralateral one, and it was difficult to match the characteristics between the healthy group and patients. Therefore, this could be a selection bias and individual walking variations could have potentially affected the outcomes. Last but not least, the retrospective design of the current study made the patient selection a confounding factor. 


\section{Conclusion}

After TKA, patients have altered gait kinematics compared with the control group. MR knees showed less adduction/abduction ROM and proximal/distal translation, while SR knees had smaller medial/lateral translation. With regard to the gait curve, both TKA knees showed more extension during stance phase. MR knees presented a varied rotating mode opposed to normal knees, and they also showed more medial/lateral displacement. SR knees partly restored the mode in internal/external rotation and proximal/distal translation when compared with normal knees, but they showed more abducted during the stance phase and more medial translated in midstance and mid-swing phase. These deviated characteristics in gait kinematics may be the reason for the difference in functional outcome.

\section{Acknowledgments}

We thank the volunteers who participated in this study.

\section{Authors' contributions}

Study design: ML, WH. Administrative support: YZ, YM, QZ. Surgery performance: YM. Study conduct: ML, GF. Data collection: ML, RZ. Data analysis and data interpretation: $M L, B L, W H$. Drafting manuscript: ML. Revising manuscript content: ML. Approving final version of manuscript: QZ. QZ takes responsibility for the integrity of the data analysis. The author(s) read and approved the final manuscript.

\section{Funding}

This study was supported by the Frontier and Key Technologies Innovation Funding Project of Department of Science and Technology of Guangdong Province (No. 2015B020225007), the Program of Science and Technology of Guangzhou (201904010424) and the Medical Scientific Research Foundation of Guangdong Province (grant number A2020023).

\section{Availability of data and materials}

The data and materials used and/or analyzed during the current study are not publicly available but available from the corresponding author on reasonable request.

\section{Ethics approval and consent to participate}

The present study was approved by the institutional review board of Guangdong Provincial People's Hospital and signed informed consent for participation was obtained from all study patients.

\section{Consent for publication}

Signed informed consent for publication was obtained from all authors

\section{Competing interests}

The authors declare that they have no competing interests.

\section{Author details}

${ }^{1}$ Division of Joint Osteopathy and Traumatology, Center of Orthopedics Surgery, Guangdong Provincial People's Hospital, Guangdong Academy of Medical Sciences, School of Medicine, South China University of Technology, 106 Zhongshaner Road, Yuexiu District, Guangzhou 510080, PR China.

${ }^{2}$ Shantou University Medical College, Shantou 515063, PR China.

Received: 12 March 2020 Accepted: 16 June 2020

Published online: 04 July 2020

\section{References}

1. Skou ST, Roos EM, Laursen MB, et al. A randomized, controlled trial of Total knee replacement. N Engl J Med. 2015;373(17):1597-606.

2. Carr AJ, Robertsson O, Graves S, et al. Knee replacement. Lancet. 2012 379(9823):1331-40.
3. Inacio MCS, Paxton EW, Graves SE, et al. Projected increase in total knee arthroplasty in the United States - an alternative projection model. Osteoarthr Cartil. 2017;25(11):1797-803.

4. Hamilton DF, Lane JV, Gaston $P$, et al. What determines patient satisfaction with surgery? A prospective cohort study of 4709 patients following total joint replacement. BMJ Open. 2013;3(4):e002525.

5. Bourne RB, Chesworth BM, Davis AM, et al. Patient satisfaction after total knee arthroplasty: who is satisfied and who is not? Clin Orthop Relat Res. 2010;468(1):57-63.

6. Cao S, Liu N, Han W, et al. Simplified Chinese version of the forgotten joint score (FJS) for patients who underwent joint arthroplasty: cross-cultural adaptation and validation. J Orthop Surg Res. 2017;12(1):6.

7. Frankel $\mathrm{VH}$, Burstein $\mathrm{AH}$, Brooks DB. Biomechanics of internal derangement of the knee. Pathomechanics as determined by analysis of the instant centers of motion. J Bone Joint Surg Am. 1971;53(5):945-62.

8. Eckhoff D, Hogan C, DiMatteo L, et al. Difference between the epicondylar and cylindrical axis of the knee. Clin Orthop Relat Res. 2007;461:238-44.

9. Hollister AM, Jatana S, Singh AK, et al. The axes of rotation of the knee. Clin Orthop Relat Res. 1993;290:259-68.

10. Li M, Deng $Z$, Jiang $H$, et al. Single radius versus multiple radius femoral prostheses in total knee arthroplasty: a meta-analysis. Int J Clin Exp Med. 2019;12(8):9508-21.

11. Larsen B, Jacofsky MC, Jacofsky DJ. Quantitative, comparative assessment of gait between single-radius and multi-radius Total knee Arthroplasty designs. J Arthroplast. 2015;30(6):1062-7.

12. Cook LE, Klika AK, Szubski CR, et al. Functional outcomes used to compare single radius and multiradius of curvature designs in total knee arthroplasty. J Knee Surg. 2012;25(3):249-53.

13. Gomez-Barrena E, Fernandez-Garcia C, Fernandez-Bravo A, et al. Functional performance with a single-radius femoral design total knee arthroplasty. Clin Orthop Relat Res. 2010;468(5):1214-20.

14. Collados-Maestre I, Lizaur-Utrilla A, Gonzalez-Navarro B, et al. Better functional outcome after single-radius TKA compared with multi-radius TKA. Knee Surg Sports Traumatol Arthrosc. 2017;25(11):3508-14.

15. Tamaki M, Tomita T, Yamazaki T, et al. Factors in high-flex posterior stabilized fixed-bearing total knee arthroplasty affecting in vivo kinematics and anterior tibial post impingement during gait. J Arthroplast. 2013;28(10): 1722-7.

16. Li M, Zhang $L$, Zhang $R$, et al. Better quadriceps and hamstring strength is achieved after total knee arthroplasty with single radius femoral prostheses: a retrospective study based on isokinetic and isometric data. Arthroplasty. 2020;2(1):5

17. Zhang $Y$, Huang W, Yao Z, et al. Anterior cruciate ligament injuries Alter the kinematics of knees with or without meniscal deficiency. Am J Sports Med. 2016:44(12):3132-9.

18. Zhang Y, Yao Z, Wang S, et al. Motion analysis of Chinese normal knees during gait based on a novel portable system. Gait Posture. 2015;41(3):763-8.

19. Yan $W, X u X, X u Q$, et al. In vivo gait kinematics of the knee after anatomical and non-anatomical single-bundle anterior cruciate ligament reconstruction-a prospective study. Ann Transl Med. 2019;7(24):799.

20. Braito M, Giesinger JM, Fischler S, et al. Knee extensor strength and gait characteristics after minimally invasive Unicondylar knee Arthroplasty vs minimally invasive Total knee Arthroplasty: a nonrandomized controlled trial. J Arthroplast. 2016;31(8):1711-6.

21. Wang $H$, Simpson KJ, Ferrara MS, et al. Biomechanical differences exhibited during sit-to-stand between total knee arthroplasty designs of varying radii. J Arthroplast. 2006;21(8):1193-9.

22. Mahoney OM, McClung CD, dela Rosa MA, et al. The effect of total knee arthroplasty design on extensor mechanism function. J Arthroplast. 2002; 17(4):416-21.

23. Shabani B, Bytyqi D, Lustig $S$, et al. Gait changes of the ACL-deficient knee 3D kinematic assessment. Knee Surg Sports Traumatol Arthrosc. 2015;23(11): 3259-65.

24. Wang $\mathrm{H}$, Simpson $\mathrm{KJ}$, Chamnongkich S, et al. A biomechanical comparison between the single-axis and multi-axis total knee arthroplasty systems for the stand-to-sit movement. Clin Biomech (Bristol, Avon). 2005;20(4):428-33.

25. Tamaki M, Tomita T, Yamazaki T, et al. In vivo kinematic analysis of a highflexion posterior stabilized fixed-bearing knee prosthesis in deep kneebending motion. J Arthroplast. 2008;23(6):879-85.

26. Freeman MA, Pinskerova $\mathrm{V}$. The movement of the normal tibio-femoral joint. J Biomech. 2005;38(2):197-208. 
27. Komistek RD, Dennis DA, Mahfouz M. In vivo fluoroscopic analysis of the normal human knee. Clin Orthop Relat Res. 2003;410:69-81.

28. Kessler O, Durselen L, Banks S, et al. Sagittal curvature of total knee replacements predicts in vivo kinematics. Clin Biomech (Bristol, Avon). 2007. 22(1):52-8.

29. Stoddard JE, Deehan DJ, Bull AM, et al. The kinematics and stability of single-radius versus multi-radius femoral components related to mid-range instability after TKA. J Orthop Res. 2013;31(1):53-8.

30. Catani F, Fantozzi S, Ensini A, et al. Influence of tibial component posterior slope on in vivo knee kinematics in fixed-bearing total knee arthroplasty. J Orthop Res. 2006;24(4):581-7.

31. Sumner B, McCamley J, Jacofsky DJ, et al. Comparison of Knee Kinematics and Kinetics during Stair Descent in Single- and Multi-Radius Total KneeArthroplasty. J Knee Surg. 2019. https://doi.org/10.1055/s-0039-1692652.

32. Khasian M, Sharma A, Fehring TK, et al. Kinematic performance of gradually variable radius posterior-stabilized primary TKA during various activities: an in vivo study using fluoroscopy. J Arthroplast. 2020;35(4):1101-8.

\section{Publisher's Note}

Springer Nature remains neutral with regard to jurisdictional claims in published maps and institutional affiliations.

Ready to submit your research? Choose BMC and benefit from:

- fast, convenient online submission

- thorough peer review by experienced researchers in your field

- rapid publication on acceptance

- support for research data, including large and complex data types

- gold Open Access which fosters wider collaboration and increased citations

- maximum visibility for your research: over $100 \mathrm{M}$ website views per year

At $\mathrm{BMC}$, research is always in progress.

Learn more biomedcentral.com/submissions 